\title{
Mitochondrial membrane permeabilization produced by PTP, Bax and apoptosis: a ${ }^{1} \mathrm{H}-\mathrm{NMR}$ relaxation study
}

\author{
D Pouliquen ${ }^{\star, 1}$, G Bellot ${ }^{1}$, G Guihard ${ }^{1}$, P Fichet ${ }^{1}$, K Meflah $^{1}$ and \\ FM Vallette ${ }^{1}$ \\ 1 Inserm UMR 601, Equipe 4, Institut de Biologie, 9 Quai Moncousu, 44035 \\ Nantes cédex, France \\ * Corresponding author: D Pouliquen, Inserm UMR 601, Equipe 4, Institut de \\ Biologie, 9 Quai Moncousu, 44035 Nantes cédex, France. \\ Tel: + 33-2-40-08-41-96; Fax: + 33-2-40-08-40-82; \\ E-mail: daniel.pouliquen@univ-nantes.fr
}

Received 24.1.05; revised 16.5.05; accepted 16.6.05; published online 29.7.05 Edited by JC Martinou

\begin{abstract}
To analyze the involvement of structured water (bound to macromolecules) in apoptosis-induced mitochondrial outermembrane permeability, we compared the dynamics of water protons from nuclear magnetic resonance (NMR) data in apoptotic liver mitochondria with that of control mitochondria incubated in vitro with free $\mathrm{Ca}^{2+}$ (opening of the permeability transition pore, PTP) or with Bax $\alpha$. Our results demonstrate that water molecules in apoptotic mitochondria exhibit an accelerated translational motion of structured water common with that induced by the opening of the PTP, but limited in amplitude. On the other hand, no significant quantitative change in structured water was observed in apoptotic mitochondria, a phenomenon also observed with Bax $\alpha$ induced permeability. We conclude that the changes observed in the different water phases differ both quantitatively and qualitatively during the opening of the PTP and the Bax $\alpha$-induced permeability, and that the apoptotic mitochondria exhibit mixed properties between these model situations. Cell Death and Differentiation (2006) 13, 301-310.

doi:10.1038/sj.cdd.4401731; published online 29 July 2005
\end{abstract}

Keywords: apoptosis in vivo; mitochondria; water; permeability transition; $\operatorname{Bax} \alpha$

Abbreviations: $\mathrm{HCM}$, heavy fraction of control mitochondria; HAM, heavy fraction of apoptotic mitochondria; NMR, nuclear magnetic resonance; PTP, permeability transition pore

\section{Introduction}

The mitochondrion, conventionally considered the cell's powerhouses, is intimately entwined in apoptosis. ${ }^{1}$ In particular, a mitochondria-dependent step, involving outer-membrane permeabilization, appears related with most proapoptotic stimuli. This process is controlled by the $\mathrm{Bcl}-2$ family of proteins, ${ }^{2}$ as activation members of the proapoptotic subgroup of this protein family, $\operatorname{Bax} \alpha,{ }^{3}$ lead to the cytosolic release of mitochondrial intermembrane space proteins. The mechanism by which these proteins induce the release of mitochondrial proteins is still discussed. One proposed model involves rupture of the mitochondrial outer membrane as a consequence of mitochondrial swelling after the opening of the permeability transition pore (PTP). ${ }^{4,5}$ According to another model, proapoptotic $\mathrm{Bcl}-2$ proteins like Bax induce a selective process of outer-membrane permeabilization independent of the PTP, ${ }^{6}$ through the formation of channels or pores, ${ }^{7}$ allowing the selective release of proteins soluble in the innermembrane space such as cytochrome $c$.

Among the different biophysical techniques allowing the characterization of mitochondrial outer-membrane permeability, proton nuclear magnetic resonance $\left({ }^{1} \mathrm{H}-\mathrm{NMR}\right)$ is of great interest as it provides information on the interactions between protons from individual solvent molecules and protons from protein structures. ${ }^{8}$ In particular, measurements of the ${ }^{1} \mathrm{H}$-NMR relaxation times over a wide temperature range allow the changes in rotational and diffusional water dynamics to be determined. ${ }^{9}$ In addition, these informations can be obtained in a nondestructive manner, allowing final confrontation of the data successively recorded by different techniques on the very same biological samples.

Using the water molecule as a sensor of the physicochemical properties inside the cell, Hortelano et al. ${ }^{10}$ performed ${ }^{1} \mathrm{H}$ NMR analysis of the intracellular medium during apoptosis. They showed that intracellular T2 and the apparent selfdiffusion of intracellular water decreased significantly after caspase activation in macrophages, indicating slower rotational and translational motions of water during apoptosis. ${ }^{10}$ Preliminary investigations have also demonstrated a high level of structuration of water in mitochondria, which suggests the existence of a complex heterogeneous distribution of mitochondrial water in different phases. ${ }^{11}$ Also, the water proton relaxation times of mitochondria isolated from ischemic hearts are decreased compared with their normal counterparts. ${ }^{12}$ This latter observation suggests that NMR relaxation studies represent a nondestructive mean of physical characterization of mitochondrial water, in relation with structural and/or functional changes in pathological situations. To date, NMR investigations of the dynamics and environment of water in suspensions of isolated rat liver mitochondria have demonstrated a considerable viscosity of the mitochondrial matrix, ${ }^{13}$ which is in good agreement with an important crowding and the presence of multienzyme complexes. ${ }^{14}$ However, in the conditions generally used for relaxation time measurements of total water in suspensions of mitochondria (room temperature, medium-to-high field strength), the fast water exchange observed across the inner mitochondrial membrane and an intramitochondrial water residence time of about $100 \mu \mathrm{s},{ }^{12,13}$ mean that the mean distance span by bulk water molecules (i.e. osmotically active) exceeds the mean dimensions of these organelles by a factor of $20-100 .{ }^{15}$ Other complementary NMR techniques such as ${ }^{17} \mathrm{O}$ magnetic resonance spectroscopy have confirmed the existence of a highly structured water phase (osmotically inactive) in 
mitochondria. ${ }^{16}$ However, this approach only provides information on the $1-2 \%$ of the total matrix water volume, which present mean rotational correlation times of the order of $10^{-6} \mathrm{~s}$, corresponding to the strongly bound water phase.

Recent work has confirmed that investigations on the involvement of mitochondria in apoptosis contribute to elucidate the mechanisms by which the release of apoptogenic factors can be modulated. ${ }^{17}$ In this study, a new method of characterization of mitochondrial water was used, which takes into account the importance of magnetic crossrelaxation between protein protons and water protons, ${ }^{18}$ based on the analysis of the temperature dependency of proton relaxation times of both bulk and structured water on a low-field $(20 \mathrm{MHz})$ NMR spectrometer. This technique allows the characterization of the physical changes observed in the whole structured water fraction (the totality of water that does not freeze under the freezing point of bulk water) to be determined. ${ }^{19,20}$ The experiments contribute to give a new insight in the mechanism of mitochondrial membrane permeabilization produced by apoptosis in vivo compared with the effect of $\operatorname{Bax} \alpha$ and the opening of the PTP induced by $\mathrm{Ca}^{2+}$ in vitro.

\section{Results}

Mitochondria contain a high proportion of structured water, which can be analyzed by measuring physical parameters illustrated in Figure 1. We thus use ${ }^{1} \mathrm{H}$-NMR to analyze the effect of outer-membrane permeabilization on water both in model situation and during in vivo apoptosis.

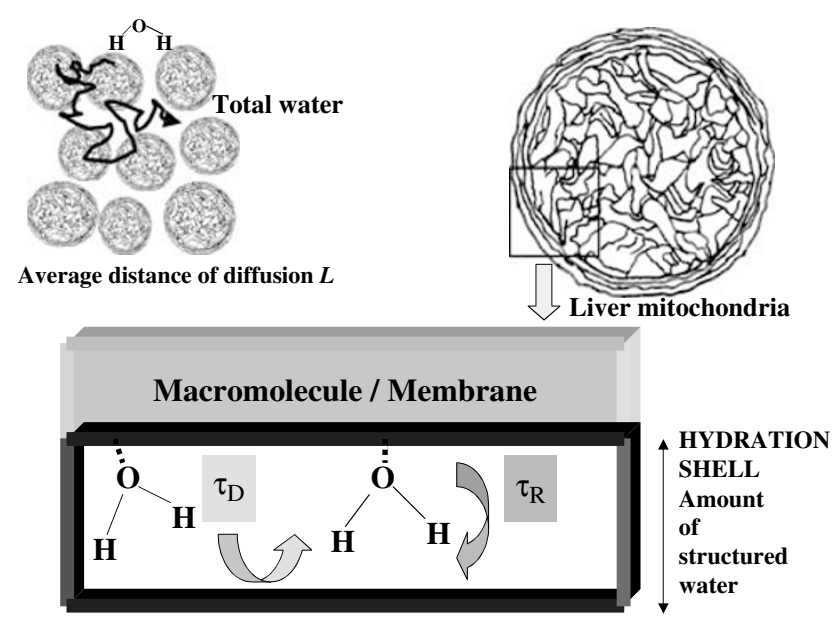

Figure 1 A schematic illustration of the various dynamic processes experienced by water molecules in liver mitochondria. Above the freezing point (top left), water molecules free of the influence of the macromolecules tumble and diffuse over a long distance $L$ during a relaxation time measurement (Tlobs or T2obs). Below the freezing point $\left(\theta<-15^{\circ} \mathrm{C}\right)$, the NMR signal is restricted to water molecule protons interacting with macromolecules and membrane surfaces (structured water) within a mitochondrion (top right). Quantitatively, changes in the amount of structured water may occur within the hydration shell of macromolecules. Structured water molecules tumble anisotropically, this process being represented by specific correlation times $\tau_{\mathrm{D}}$ (diffusion from one region to another) and $\tau_{\mathrm{R}}$ (rotational motion). Their temperature dependence is given by the Arrhenius relation: $\tau_{(\lambda)}=\tau^{0} \exp (E / k T)$, where $E$ is the activation energy, $\tau^{0}$ the preexponential factor, $k$ the Boltzmann constant and $T$ the absolute temperature

\section{Relaxation process in control mitochondria pellets}

The water relaxation times observed at $4{ }^{\circ} \mathrm{C}$ in mitochondria pellets are determined by the decay of magnetically labelled water in the intramitochondrial space, in the external medium and by the exchange of magnetically labelled water between these two compartments. ${ }^{13}$ The mean Tlobs of the mitochondria pellet at $4{ }^{\circ} \mathrm{C}$ was $286 \pm 6 \mathrm{~ms}$ (monoexponential fitting), and values of T2obs measurements with mono- and biexponential fitting were $59.5 \pm 0.2 \mathrm{~ms}$ (amplitude $=100.5 \pm$ 0.2 ), and $20 \pm 10 \mathrm{~ms}$ (amplitude $a=2 \pm 1$ ) and $66.1 \pm 0.8 \mathrm{~ms}$ (amplitude $b=99 \pm 1$ ), respectively (Table S1 and Figure S1). Then, the absence of significant second component in the relaxation behavior suggested a fast exchange rate between the intramitochondrial space and the external medium. Relaxation time measurements in the presence of superparamagnetic nanoparticles revealed a higher relaxation enhancement of bulk water in the supernatant compared with that in the mitochondria pellet (Table S1). Below the freezing transition, this relaxation enhancement was considerably reduced at $258 \mathrm{~K}$ and disappeared at $249 \mathrm{~K}$ for mitochondria, in good agreement with the dramatic reduction of the exchange process between structured water in mitochondria and in the external medium (Table S2).

The concentration of sugars in the mitochondria pellet was $13.1 \mathrm{~g} / \mathrm{l}$ while $48.0 \mathrm{~g} / \mathrm{l}$ in the external medium (supernatant at $8700 \times g$ ). This result means that the final concentration in mannitol would be $0.141 \mathrm{M}$, while that of water is of the order of $55.5 \mathrm{M} \times 75 \%$ (mean water content $)=41.6 \mathrm{M}$. Given the water molecule contains two protons and the mannitol 14, this leads to a final ratio of protons from water versus those from mannitol of 42 in the mitochondria. In conclusion, nonwater protons do not appear to significantly contribute to the NMR signal detected.

\section{Apoptosis induced in vivo}

Mitochondria were purified through density gradient. The results reported below correspond to data collected mainly on the heavy fractions of control (HCM) or apoptotic mitochondria (HAM), at the $1.051 / 1.096 \mathrm{~g} / \mathrm{ml}$ density interface. ${ }^{21}$ Electron micrographs of heavy and light liver mitochondria samples are shown in Figure 2a. High magnifications revealed that HAM and $\mathrm{HCM}$ exhibit conventional crest-forming inner membrane, which did not differ in size. However, HAM exhibited some increase in the electron lucency of the matrix compared with HCM. In heavy apoptotic mitochondria, cytochrome $c$ was released (Figure $2 \mathrm{~b}$ ) and a very significant increase in the T2obs was observed compared with control (Figure $3 a$ and Table S3, $0.001<P<0.01)$. In contrast, the increase in the T1obs was slight. Previous data have also shown that Bax was mainly present as a monomer in HAM population. ${ }^{21}$ Light control mitochondria differ from $\mathrm{HCM}$ by a significant increase in the values of T1obs and T2obs $(+50 \%$ and $+200 \%$, respectively). However, no significant difference was observed between NMR parameters of the light fraction of apoptotic (LAM) liver mitochondria compared with control (LCM). The temperature dependency of the characteristic 'average' time $\tau_{\mathrm{D}}$ between two molecular orientations for structured water molecules undergoing translational motion 
a

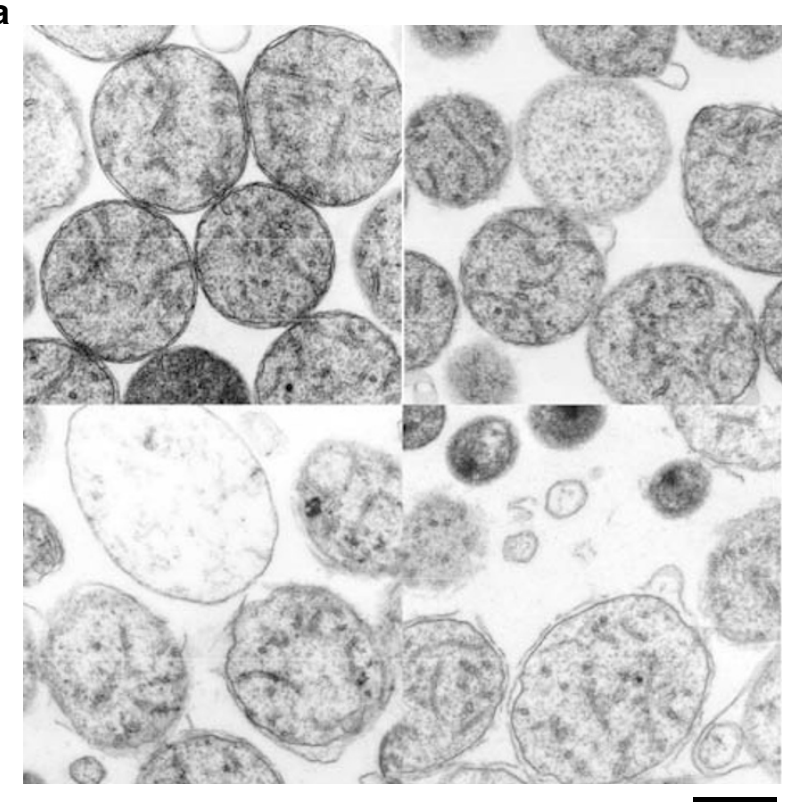

b

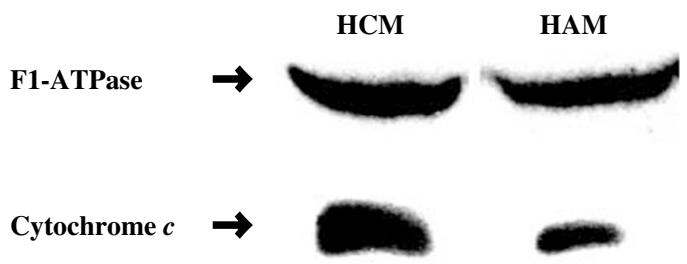

Figure 2 Morphological and biochemical characterization of apoptotic mitochondria. (a) Electron micrographs of control (left column) and apoptotic (right column) liver mitochondria. Heavy fraction (HCM and HAM), top. Light fraction (LCM and LAM), bottom. The scale bar corresponds to $0.5 \mu \mathrm{m}$. (b) Immunoblot analysis of the effect of apoptosis in vivo on the concentration on mitochondrial intermembrane proteins. The levels were determined by Western blotting of isolated mitochondrial proteins with antibodies against cytochrome $c$ and FI-ATPase $\beta$-subunit

(Figure 1) showed a pattern (Figure $3 \mathrm{~b}$ and Table S4) comparable to that of mitochondria with a moderately opened PTP induced by $100 \mu \mathrm{M} \mathrm{Ca}^{2+}$ (Figure $6 \mathrm{~b}$ and Table S6). However, comparison of the amount of structured water between apoptotic (HAM) and control mitochondria (HCM) revealed a slight decrease in the whole temperature range together with a tendency towards an increase in the slope of the temperature dependency of the quantity of structured water (Figure 3c and Table S7).

In conclusion, the relative quantity of structured water is decreased in HAM versus $\mathrm{HCM}$, and its propency to freeze is increased below $-20^{\circ} \mathrm{C}$, meaning that the degree of structuration of this water phase is less in apoptotic mitochondria, compared with control.

\section{Modelization of apoptotic outer-membrane pemeabilization}

To test whether this change in structured water is related to one of the two proposed models of mitochondrial membrane a

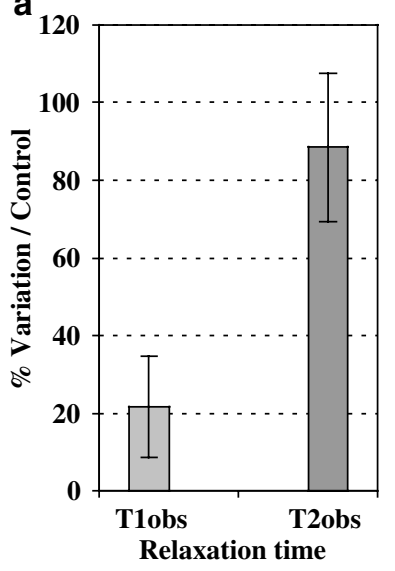

b
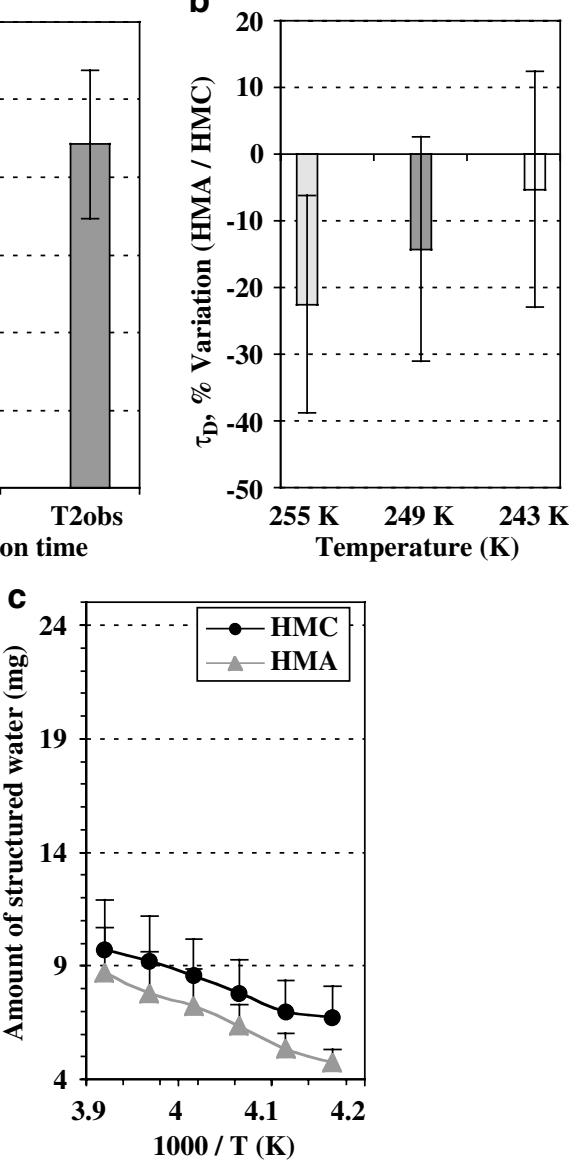

Figure 3 NMR parameters of water in apoptotic and control mitochondria. (a) Variation of relaxation times of total water in apoptotic mitochondria (HAM versus $\mathrm{HCM}$ ). Measurements performed at $4^{\circ} \mathrm{C}$ and $v=20 \mathrm{MHz}$. Data correspond to the mean \pm S.D. of three independent experiments. (b) Variation of the translational movement of structured water at three temperatures. Data correspond to the mean + S.D. of three independent experiments. (c) Amount of structured water and evolution with temperature. Data given for a constant mass $(100 \mathrm{mg})$ of frozen mitochondria pellet. Data correspond to the mean \pm S.D. of five independent experiments

permeabilization, we investigate the modifications observed in the dynamics of water phases in liver mitochondria incubated with free $\mathrm{Ca}^{2+}$ or $\mathrm{Bax} \alpha$. Figure 4 illustrates the effect of increasing concentration of free $\mathrm{Ca}^{2+}(\mathrm{a}, \mathrm{b})$ or $\mathrm{Bax} \alpha$ (c) on the mitochondrial content in cytochrome $c$ and Smac/ Diablo. In the first situation, the quantity of the two mitochondrial proteins presented a comparable exponential decrease as a function of free $\mathrm{Ca}^{2+}$ concentration. In the second situation, the decrease in quantity of cytochrome $c$ was similar to that induced by free $\mathrm{Ca}^{2+}$, and a linear relationship was observed between this drop and Bax $\alpha$ concentration on a log-log scale. In contrast, in the same conditions, the quantity of Smac/Diablo presented a slight decrease only in the 0.03 to $0.3 \mathrm{nM}$ concentration range of $\operatorname{Bax} \alpha$.

\section{Opening of the PTP in vitro}

In a first set of experiments, we confirmed $\mathrm{Ca}^{2+}$-induced mitochondrial swelling reported by others. Increasing free 
a

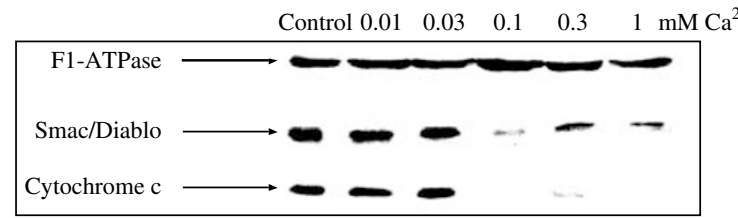

b
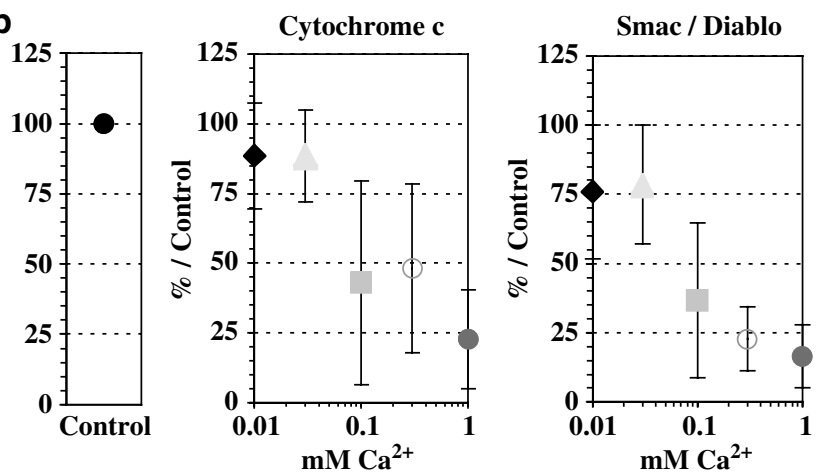

C
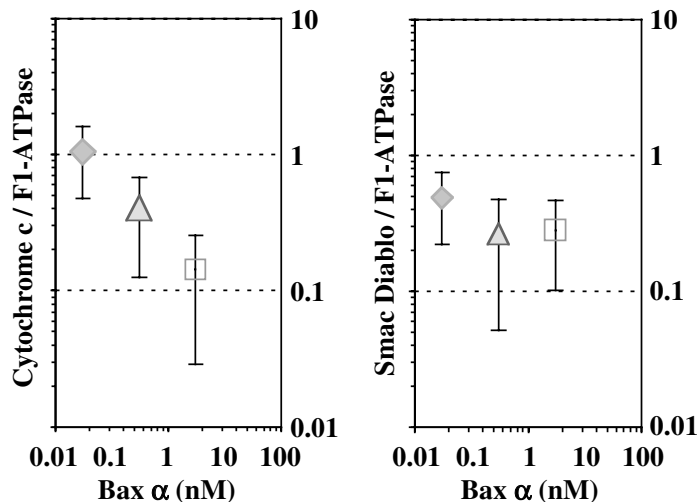

Figure 4 Effect of free $\mathrm{Ca}^{2+}(\mathbf{a}, \mathbf{b})$, or $\mathrm{Bax} \alpha$ (c) on the concentration on mitochondrial intermembrane proteins. The levels were determined by Western blotting of isolated mitochondrial proteins with antibodies against cytochrome $c$ and Smac/Diablo. (a) Immunoblot analysis of the effect of increasing concentration in free $\mathrm{Ca}^{2+}$ on the level of intermembrane proteins. (b) Evolution of the ratio of the cytochrome $c$ and Smac/Diablo content to that of FI-ATPase (comparison with control $=100 \%$ ) as a function of free $\mathrm{Ca}^{2+}$ concentration. (c) Evolution of the ratio of the cytochrome $c$ and Smac/Diablo content to that of FIATPase as a function of $\operatorname{Bax} \alpha$ concentration

$\mathrm{Ca}^{2+}$ concentrations promoted a mitochondrial swelling (Figure 5a) when compared to control condition. It was also confirmed that $\mathrm{Ca}^{2+}$-induced mitochondrial swelling could be inhibited by the pretreatment of the mitochondrial suspension with cyclosporin A, a well-known inhibitor of PTP. Interestingly, the higher the free $\mathrm{Ca}^{2+}$ concentration was raised, the larger the extent of mitochondrial swelling was observed (Figure 5a). Such an extent was plotted as a function of free $\mathrm{Ca}^{2+}$ concentration. As shown in Figure 5b, there was a clear relationship between free $\mathrm{Ca}^{2+}$ concentration and osmotic swelling that could be fitted by a Hill-like equation from which an $\mathrm{EC}_{50} \sim 0.85 \mathrm{mM}$ was calculated. These data established that mitochondria incubated in our conditions have functional PTP, as its opening is stimulated by $\mathrm{Ca}^{2+}$ and inhibited by cyclosporin A.

The response of water proton NMR parameters of the dense fraction of liver mitochondria (HMC) to increasing a

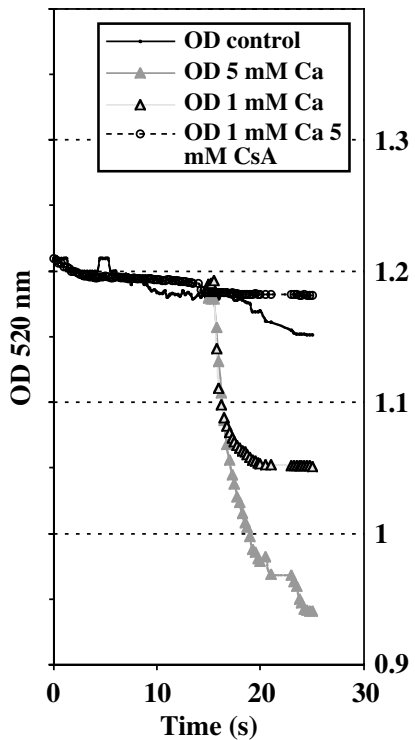

b

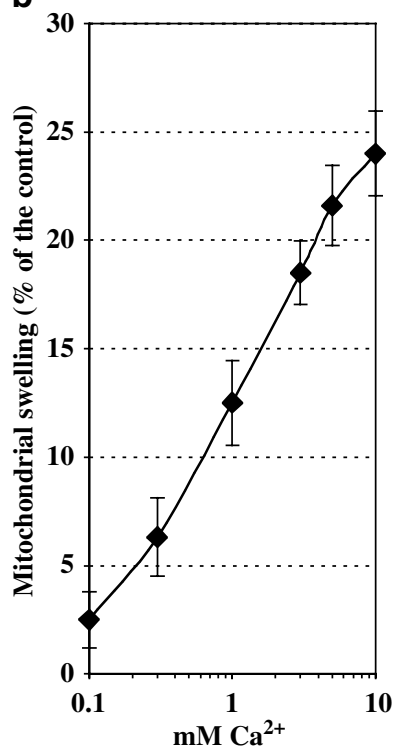

Figure $5 \mathrm{Ca}^{2+}$-induced mitochondrial osmotic swelling. Purified mitochondria were incubated $(0.2 \mathrm{mg}$ protein $/ \mathrm{ml})$ as described in Materials and Methods. (a) The optical density (measured at $520 \mathrm{~nm}$ ) of the suspension was monitored in the absence or the presence of $5 \mathrm{mM}$ cyclosporin $\mathrm{A}$, in control condition and after the addition of 5 or $1 \mathrm{mM}$ free $\mathrm{Ca}^{2+}$. All the traces are representative of three independent experiments. (b) The osmotic swelling was deduced from the $O D^{520}$ variation $10 \mathrm{~min}$ after $\mathrm{Ca}^{2+}$ load and was expressed as a percentage of $O D^{520}$ value measured just before $\mathrm{Ca}^{2+}$ addition. The result was plotted as a function of free $\mathrm{Ca}^{2+}$ concentration. Data correspond to the mean \pm S.E.M. of three independent experiments and their distribution was fitted by using a pseudo-Hill equation from which an $\mathrm{EC}_{\mathrm{SO}} 0.85 \mathrm{mM}$ and a Hill number close to 1 were deduced

doses of free $\mathrm{Ca}^{2+}$ is shown in Figure 6 . The results indicate that the relaxation times of bulk water are significantly increased when free $\mathrm{Ca}^{2+}$ was above $100 \mu \mathrm{M}$ (significance: $0.02<P<0.05$ for T1obs, $0.001<P<0.01$ for T2obs). Both T1obs and T2obs present a dramatic rise between 30 and $100 \mu \mathrm{M} \mathrm{Ca}{ }^{2+}$. However, the relaxation times increase between control and $1 \mathrm{mM} \mathrm{Ca}^{2+}$ was more pronounced for T2obs compared with T1obs (Figure 6a and Table S5).

Below the freezing transition, relaxation time measurements are limited to the structured water fraction as bulk water protons are technically unobservable with the spectrometer when water is frozen. Figure $6 \mathrm{~b}$ (and Table S6) shows the progressive drop in the correlation time for translational motion of structured water, at $255 \mathrm{~K}$, with regard to the free $\mathrm{Ca}^{2+}$ concentration in the medium. This decrease, particularly significant at $255 \mathrm{~K}$, tends to disappear with the decrease in temperature (no significant difference was observed between $1 \mathrm{mM} \mathrm{Ca}^{2+}$ and control at $249 \mathrm{~K}$ and below, Figure $6 \mathrm{c}$ and Table S7). Figure 6d illustrates the rise in the amount of structured water observed in HMC following incubation with increasing concentrations in free $\mathrm{Ca}^{2+}$. This rise, which was significant from $100 \mu \mathrm{M}$, was acompanied by an increase in the slope of the temperature dependency of the quantity of structured water. According to Van't Hoff law and the physical principles of NMR, an increase in the value of the correlation times for both translational $\left(\tau_{\mathrm{D}}\right)$ and rotational $\left(\tau_{\mathrm{R}}\right)$ motions of structured water is expected when the temperature 

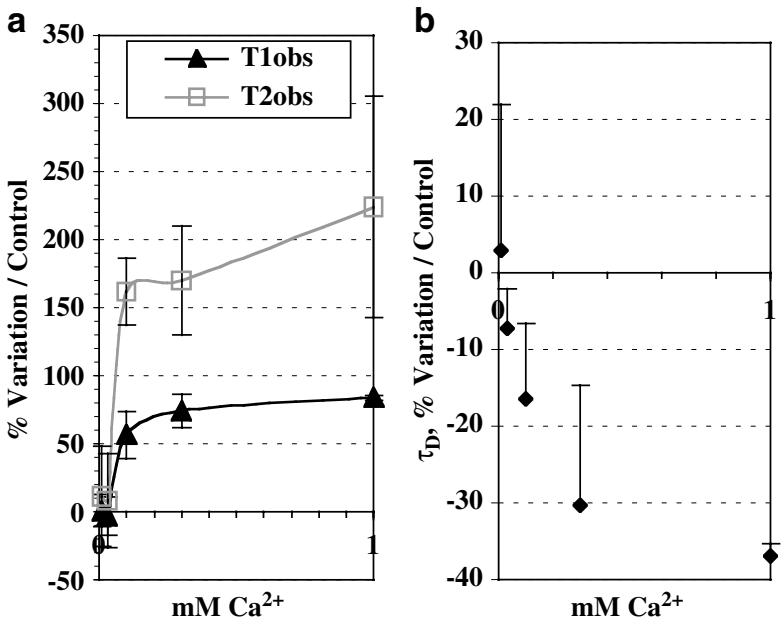

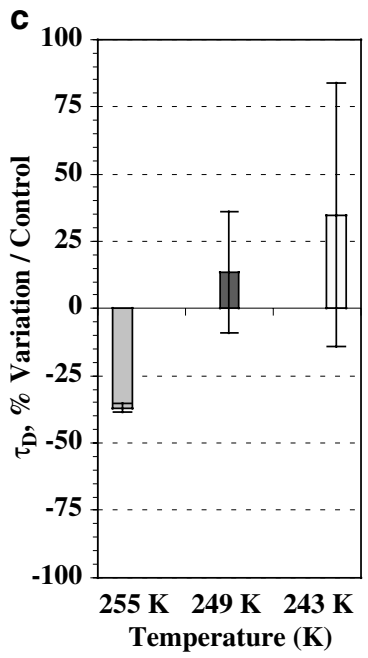

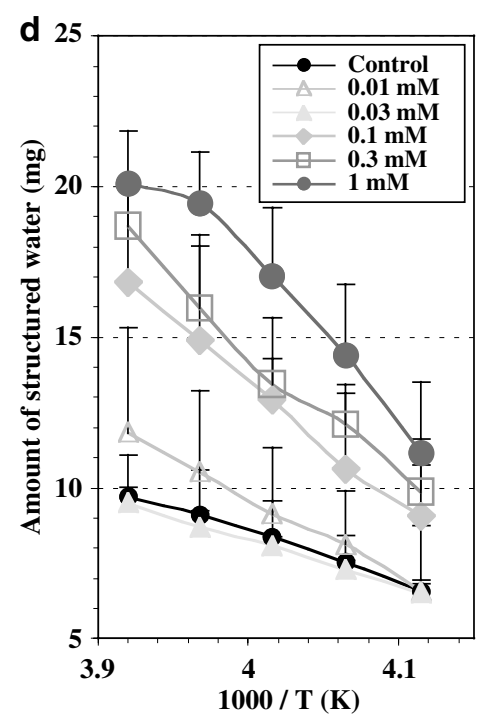

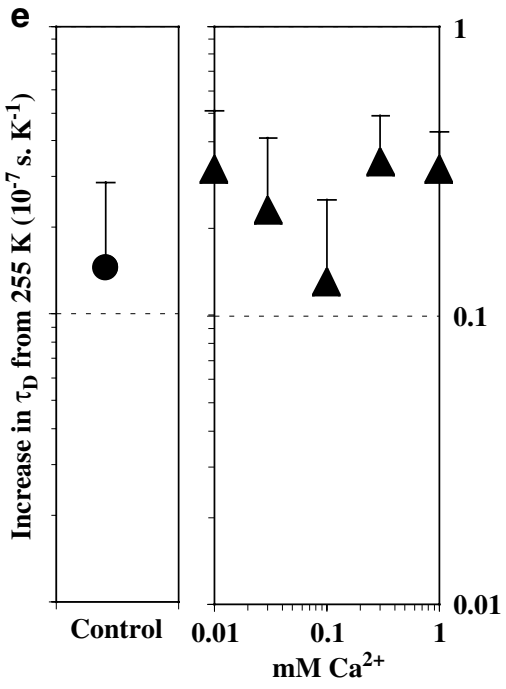

Figure 6 Effect of free $\mathrm{Ca}^{2+}$ concentration on NMR parameters of mitochondrial water. (a) Variation of the spin-lattice (T1obs) and spin-spin (T2obs) relaxation times of total mitochondrial water relative to control. Measurements performed at $4^{\circ} \mathrm{C}$ and $\mathrm{v}=20 \mathrm{MHz}$. Data correspond to the mean \pm S.D. of three independent experiments. (b) Dose dependency at $255 \mathrm{~K}$ of the variation of the translational motion of mitochondrial structured water (correlation time $\tau_{\mathrm{D}}$ ). Data correspond to the mean \pm S.D. of three independent experiments. (c) Temperature dependency of the variation in the translational motion of mitochondrial structured water (correlation time $\tau_{\mathrm{D}}$ ) for $1 \mathrm{mM}$ $\mathrm{Ca}^{2+}$. Data correspond to the mean + S.D. of three independent experiments. (d) Changes in the amount of mitochondrial structured water. The data correspond to a constant mass $(100 \mathrm{mg})$ of frozen mitochondria pellet and a constant protein content $(15 \mathrm{mg})$. Data correspond to the mean \pm S.D. of three independent experiments. (e) Dependency of the energy barrier for translational motion of mitochondrial structured water on free $\mathrm{Ca}^{2+}$ concentration. Data correspond to the mean \pm S.D. of three independent experiments

decreases. The difference observed between the values of $\tau_{\mathrm{D}}$ at 243 and $255 \mathrm{~K}$, minus that at $255 \mathrm{~K}$ (which directly depends on the absolute enthalpy of the system) represents an energy barrier for the motion of structured water. Figure $6 \mathrm{e}$ demonstrates that incubation of $\mathrm{HMC}$ with increasing concentrations in free $\mathrm{Ca}^{2+}$ did not produce any significant change in this parameter.

In conclusion, liver mitochondria with an opened PTP exhibit an acceleration of the molecular dynamics of both total mitochondrial water and its structured subfraction. This qualitative change is common, while much pronounced to that observed in HMA. In contrast, the structured water fractions differ markedly in HMA and swollen mitochondria on the quantitative viewpoint.

\section{Effect of Bax $\alpha$ in vitro}

Incubation with $3 \mathrm{nM} \operatorname{Bax} \alpha$ acellular did not produce any significant increase in the relaxation times of total water (Figure $7 \mathrm{a}$ and Table S8). The effect of $\mathrm{Bax} \alpha$ on mitochondrial structured water also presented a very different pattern when compared with that induced by the opening of the PTP. Firstly, at $255 \mathrm{~K}$ and with the highest $\mathrm{Bax} \alpha$ concentration, the decrease in $\tau_{\mathrm{D}}$ appeared not significant compared with control (Figure $7 \mathrm{~b}$ and Table S9). Secondly, decreasing the temperature resulted in an increase of the relative drop in $\tau_{D}$, which was significant from $249 \mathrm{~K}$ (Figure $7 \mathrm{~b}$ and Table S9). Comparison of the amount of structured water in mitochondria incubated with increasing $\operatorname{Bax} \alpha$ concentrations did not show 
any significant change compared with control (Figure 7c). In contrast, incubation with $1 \mathrm{mM} \mathrm{Ca}^{2+}$ showed a dramatic increase (Figure 7c), which was similar to that observed in Figure $6 \mathrm{~d}$. Whatever the concentration in $\operatorname{Bax} \alpha$ added, the slope of the temperature dependency of the quantity of structured water remained comparable to that of control (Figure 7c). Increasing the concentration in $\operatorname{Bax} \alpha$ (on a log scale) resulted in an exponential decrease in the energy
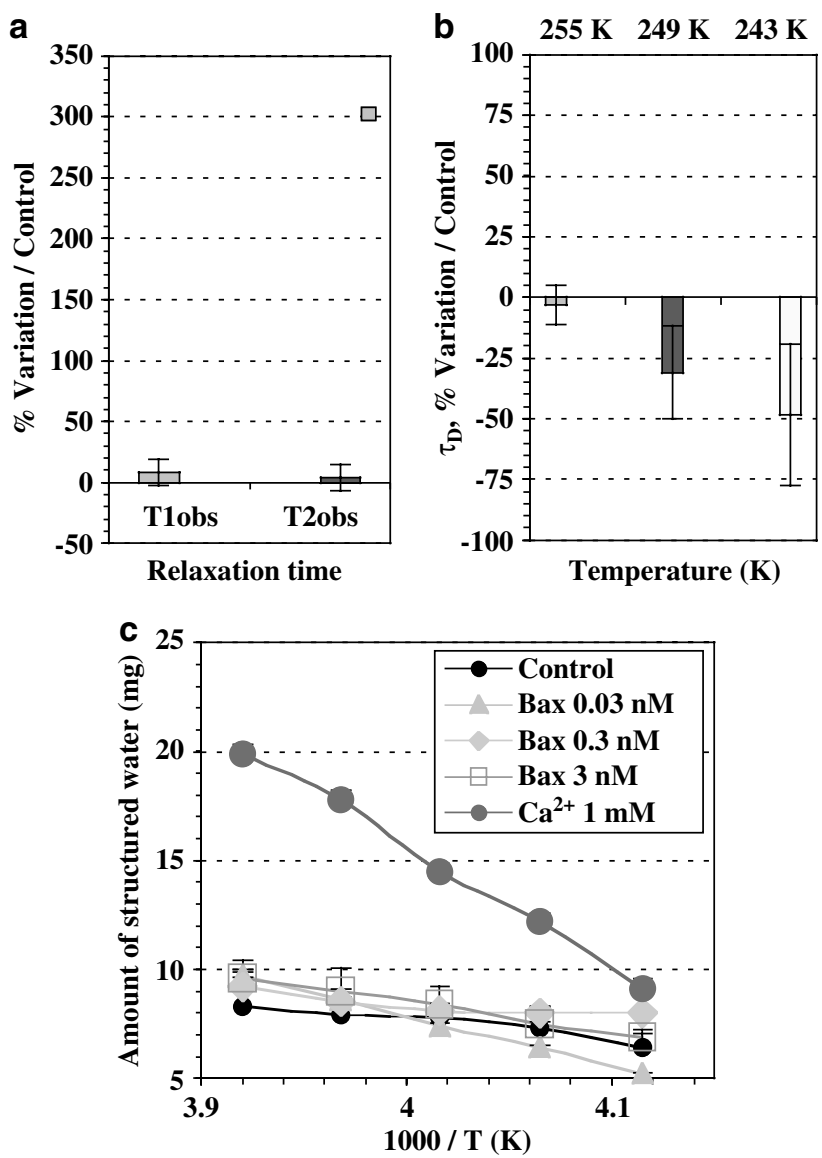

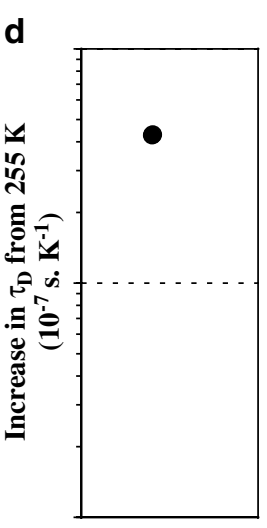

Control
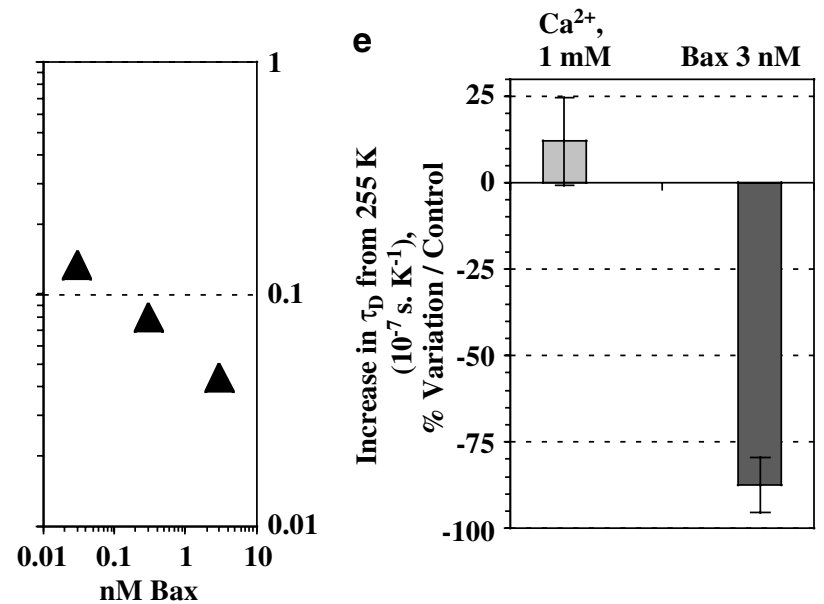

Figure 7 Effect of Bax $\alpha$ on NMR parameters of mitochondrial water. (a) Variation of the spin-lattice (T1obs) and spin-spin (T2obs) relaxation times of total mitochondrial water relative to control. Measurements performed at $4^{\circ} \mathrm{C}$ and $v=20 \mathrm{MHz}$. Data correspond to the mean \pm S.D. of three independent experiments. (b) Changes in the translational motion of mitochondrial structured water and its temperature dependency. Data correspond to the mean \pm S.D. of three independent experiments. (c) Changes in the amount of mitochondrial structured water. The data correspond to a constant mass (100 mg) of frozen mitochondria pellet. Data correspond to the mean \pm S.D. of three independent experiments. (d) Dependency of the energy barrier for the translational motion of mitochondrial structured water on Bax $\alpha$ concentration. The experiment shown is typical from three successive measurements on the same pool of mitochondria. (e) Comparison of the effect of $1 \mathrm{mM}$ $\mathrm{Ca}^{2+}$ and $3 \mathrm{nM} \mathrm{Bax} \alpha$ on the variation of the translational motion of mitochondrial structured water, relative to control, from 255 to $243 \mathrm{~K}$. Data correspond to the mean \pm S.D. of three independent experiments 
a

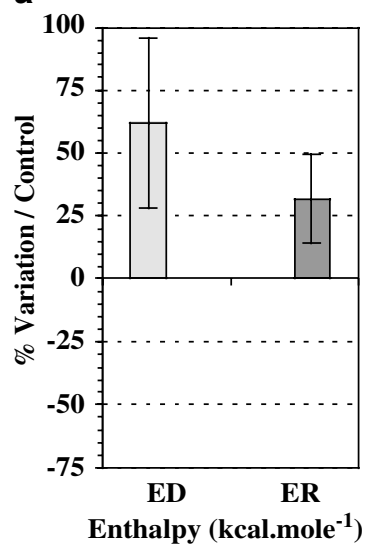

b

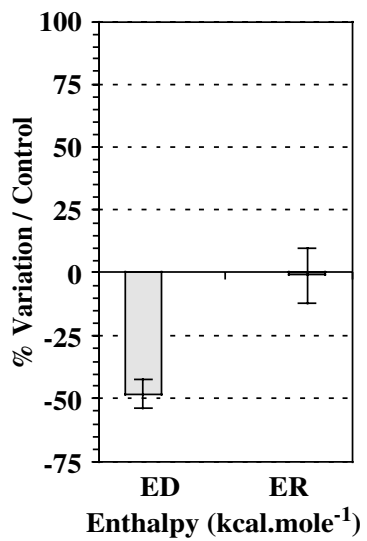

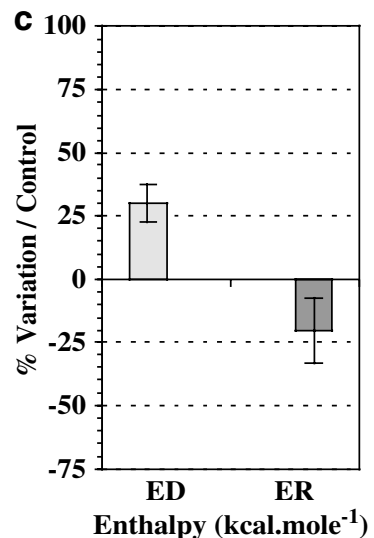

Figure 8 Comparison of the changes induced to the energies of activation for the movements of translation $\left(E_{\mathrm{D}}\right)$ and rotation $\left(E_{\mathrm{R}}\right)$ of mitochondrial structured water relative to control. (a) Effect of $1 \mathrm{mM} \mathrm{Ca}^{2+}$-induced opening of the PTP. (b) Effect of $3 \mathrm{nM} \mathrm{Bax} \alpha$. (c) Effect of apoptosis induced in vivo (HAM/HCM)

barrier to the translational movement of structured water (Figure 7d). Figure 7e shows the comparison of the effect of $1 \mathrm{mM} \mathrm{Ca}^{2+}$ and $3 \mathrm{nM} \mathrm{Bax} \alpha$ in three independent experiments, and confirms that $\operatorname{Bax} \alpha$ contributes to a dramatic decrease in this energy barrier.

Comparison of the changes in the enthalpies for the translational $\left(E_{\mathrm{D}}\right)$ and rotational $\left(E_{\mathrm{R}}\right)$ movement of structured water, relative to control, tend to confirm that apoptotic mitochondria behave like control ones with a moderately opened PTP, while Bax a present a completely different pattern (Figure 8 and Table S10).

\section{Discussion}

In apoptotic liver mitochondria, a $90 \%$ increase in the spinspin relaxation time (T2obs) of total water protons was observed at $4{ }^{\circ} \mathrm{C}$ and for a Larmor frequency of $20 \mathrm{MHz}$. This feature means that the average distance $L^{15}$ that a water molecule diffuses during a T2obs measurement increases from $11 \mu \mathrm{m}(\mathrm{HCM})$ to about $20 \mu \mathrm{m}$ (HAM), exceeding by about 12 times the mean dimension of normal rat liver mitochondria $\left(0.9 \mu \mathrm{m}^{21}\right)$. This lengthening of $L$ is far more pronounced after

complete opening of the PTP induced in vitro by free $\mathrm{Ca}^{2+}$. The corresponding 225\% increase in T2obs induced by $1 \mathrm{mM}$ $\mathrm{Ca}^{2+}$ means an important reduction of the static magnetic field inhomogeneity, which causes protons from different water molecules to precess at different frequencies, leading to phase shift differences between different molecular environments. ${ }^{15}$ These changes in the magnetic properties of mitochondrial water protons can be explained by a dramatic reduction of the viscosity of the mitochondrial matrix, which reaches 30 times that of the resuspension medium in control mitochondria. ${ }^{13}$ This change of the intramitochondrial environment is also consistent with the decrease observed in the T1obs/T2obs ratio from 8 to 5, meaning a significant reduction of the molecular crowding and molecular anisotropy. These results tend to confirm the higher sensitivity of T2obs to an increase in $\mathrm{Ca}^{2+}$ concentration. In addition, with regard to optical spectrometry, NMR appears much more sensitive as the concentration which produces half of the maximum augmentation in T2obs $\left(\mathrm{EC}_{50}\right)$ is reduced by about one order of magnitude.

With $\operatorname{Bax} \alpha$, mitochondria did not show any significant increase neither in the T2obs, nor in the T1obs, meaning that in vitro the binding of this multidomain proapoptotic protein to the outer membrane does not produce any change in the selfdiffusion coefficient of mitochondrial 'free' water.

From the determination of the temperature dependency of the T1sw and T2sw, the dynamic parameters for the structured water fraction can be calculated at the mitochondria level. In apoptotic liver mitochondria, structured water present a significant acceleration of the dynamics of translational motion evidenced by the decrease in $\tau_{D}$ and increase in $E_{\mathrm{D}}$. The same pattern is observed after opening of the PTP. However, in this latter case, the dynamics of both translational and rotational motions is affected in the same way, while in apoptotic liver mitochondria, the evolution of rotational motion singularly differs. In our experiment of PTP opening, the continuous decrease in $\tau_{D}$ observed from $10 \mu \mathrm{M} \mathrm{Ca}^{2+}$ can be explained by a reduction of the packing arrangement in space which is responsible for a progressive increase of the exchange rate between water molecules localized in different layers/molecular environments within the hydration shell of macromolecules in the matrix. ${ }^{26}$ Comparison of the temperature dependency of the amount of structured water demonstrates that the significant increase induced by $100 \mu \mathrm{M} \mathrm{Ca}^{2+}$ and over is much more at 255 than at $240 \mathrm{~K}$.

This observation means that the rise in the quantity of mitochondrial structured water, which fits well with that of T2obs, concerns mainly the more loosely bound fraction of water, which probably corresponds to the farest molecules from the macromolecules surfaces within their 'hydration shell'. As a consequence of its higher dynamics, the propency of this phase to be transformed, when the temperature decreases, into 'ice-like' water (with $\tau_{\mathrm{R}}>10^{-7} \mathrm{~s}$, and then not observed with the NMR spectrometer) is bigger. This finally contributes to a significant reduction in the amplitude of the NMR signal from 255 to $243 \mathrm{~K}$. The higher values observed in the enthalpies for both translational and rotational motions after opening of the PTP mean that the physical properties of mitochondrial structured water tends to evolve towards a phase with a looser degree of structuration. Higher enthalpy 
values are also observed for water molecules in the hydration shell of macromolecules with an open multidomain structure, in comparison with compact globular structure. ${ }^{20}$ These qualitative changes also agree with the dramatic increase observed in the amount of structured water above $30 \mu \mathrm{M}$ $\mathrm{Ca}^{2+}$

The pattern of changes in the thermodynamic properties of mitochondrial water produced by the insertion of $\operatorname{Bax} \alpha$ in the outer membrane differed by at least two points compared with that of PTP opening. Firstly, insertion of Bax $\alpha$ affected specifically structured water but not total water, as revealed by both qualitative and quantitative NMR data collected in this study. This observation demonstrates the crucial role of the former water phase in the preservation of the functional properties of mitochondria. Secondly, the mechanism of action of this proapoptotic protein involves a decrease in the activation energy of both the translational and the rotational motion of this peculiar water phase, a situation which differs from the observed increase in $E_{\mathrm{D}}$, and $E_{\mathrm{R}}$ induced by PTP opening. The dose to response study reveals that the more the concentration in Bax $\alpha$ present in the incubation medium increases, the less the energy barrier that separates these physical states is important. This observation contrasts with the absence of significant effect in mitochondria incubated with increasing concentrations of free $\mathrm{Ca}^{2+}$. As the energy required for the translational motion of molecules represent an important contribution of the total potential energy of the system, this observation means that protein conformational changes will occur more easily, according to the physical law relating the free energy difference between different protein conformations and changes in the bilayer deformation energy. $^{27}$

Then, the conclusion drawn from NMR investigations of total mitochondrial water and its structured fraction is that HAM and HCM with an opened PTP share in common a reduction of the matrix viscosity, acceleration of water dynamics and temperature dependency of its translational motion, but differ in the relative amount of structured water and physical behavior of its rotational motion. On the other hand, $\mathrm{HCM}$ incubated in vitro with $\mathrm{Bax} \alpha$ share in common a comparable level of the structured water fraction and the absence of significant change in T1obs.

Thus, this study has demonstrated through the characterization of the dynamics of the different water phases that the analysis of the temperature dependency of NMR relaxation parameters allows differentiation between global permeabilization of the mitochondrial membranes, which characterises the opening of the PTP, the insertion of proapoptotic proteins in the outer membrane and permeabilization occuring in vivo. This leads to the conclusion that in apoptosis produced in vivo, the mechanism of membrane permeabilization is much more complex that that produced by $\operatorname{Bax} \alpha$ and/or the opening of the PTP.

\section{Materials and Methods}

\section{Chemicals and antibodies}

All the salts used in this work were from analytical grades. Cyclosporin A, Escherichia coli LPS and D-galactosamine were from Sigma (St Louis, MI,
USA). The mouse lgG2b monoclonal antibodies directed against cytochrome $c$ and FIATPase $\beta$-subunit were purchased from R\&D Systems (Minneapolis, MN, USA) and Molecular Probes (Eugene, OR, USA), respectively. Anti-Smac/Diablo rabbit polyclonal IgG was from ProSci Inc. (Poway, CA, USA). The sheep secondary antibodies against rabbit $\lg G$ and mouse $\lg G$, coupled to peroxidase, were from Sanofi (France).

\section{Purification of mitochondria of normal and apoptotic mitochondria}

The purification procedure was performed according to Guihard et al. ${ }^{21}$ liver apoptosis was induced by an intraperitoneal injection of $200 \mathrm{mg}$ D-galactosamine and $10 \mathrm{mg}$ E. coli LPS as described by Galanos et al. ${ }^{22}$ After injection, the rats were left with no food but water ad libitum over a period of $5-6 \mathrm{~h}$ before killing. Livers $(7-8 \mathrm{~g})$ were homogenized with a motor-driven Teflon pestle ( 20 strokes at 103 r.p.m/min) in five volumes of homogenization buffer containing (in $\mathrm{mM}$ ): saccharose 300 and Hepes 10 $(\mathrm{pH} 7.0)$. Crude mitochondrial fraction was obtained by centrifugations at $1200 \times g(10 \mathrm{~min})$ and at $8700 \times g(10 \mathrm{~min})$ of the homogenate. Purified mitochondria were recovered by centrifugation $(40 \mathrm{~min} 9000 \times \mathrm{g})$ at the 70-30\% Percoll ${ }^{\mathrm{TM}}$ interface of a discontinuous density gradient containing $10,18,30$ and $70 \%$ Percoll $^{\mathrm{TM}}$. Purified mitochondria were washed and resuspended into $300 \mathrm{mM}$ Mannitol and $5 \mathrm{mM} \mathrm{MOPS} \mathrm{(pH} \mathrm{7.4)} \mathrm{at} 10 \mathrm{mg} / \mathrm{ml}$ protein concentration as determined by Bradford procedure using BSA as a standard. The suspension was disposed into Pyrex $10 \times 75$ heavy wall test tubes (Bibby Sterilin Lt Stone, Staffs ST15 OSA, UK), centrifuged at $8700 \times g$, and the tube containing the mitochondria pellet finally kept into liquid nitrogen after removing the supernatant.

\section{Purification of Bax $\alpha$}

The coding region of full-length $\operatorname{Bax} \alpha$ was subcloned in pDEST17 (Invitrogen, Cergy Pontoise, France) to add a His-tag at the N-terminus of the protein. His-tag Bax was expressed in bacteria and purified under denaturant conditions using an Ni-NTA affinity column (Qiagen) according to the manufacturer's instructions. Protein's refolding was performed by dialyzing the eluted fraction containing Bax against PBS for $24 \mathrm{~h}$.

\section{Electron microscopy}

Liver samples from control and treated rats were fixed in Na-cacodylate $(100 \mathrm{mM}, \mathrm{pH} 7.2)$ containing $3 \%(\mathrm{w} / \mathrm{vol}) \mathrm{OsO}_{4}$, rinsed by $300 \mathrm{mM} \mathrm{Na}-$ cacodylate $(\mathrm{pH} 7.2)$ and $64.5 \mathrm{mM} \mathrm{NaCl}$, and then deshydrated with ethanol of increasing grades. Samples were washed by propylene oxide before their inclusion in Epon 812. Ultrathin slices were stained by uranyl acetate and lead citrate. Observations were performed onto a TEM JEM 1010 (Philips).

\section{Optical spectrometry}

Osmotic swelling was monitored by optical density measurement at $520 \mathrm{~nm}$ as described previously. ${ }^{23}$ In brief, mitochondria were incubated $\left(500 \mu \mathrm{g}\right.$ protein. $\left.\mathrm{ml}^{-1}\right)$ at room temperature in $250 \mathrm{mM}$ sucrose, $80 \mathrm{mM}$ $\mathrm{KCl}$, K-malate $10 \mathrm{mM}$, K-succinate $8 \mathrm{mM}$, ATP- $-\mathrm{Na}_{2} 1 \mathrm{mM}$, EDTA-Na $1 \mathrm{mM}$, MOPS-K $20 \mathrm{mM}$ (pH 7.4) in the absence of $\mathrm{MgCl}_{2}$. PTP opening was induced by raising the free $\mathrm{Ca}^{2+}$ concentration with known volumes of $\mathrm{CaCl}_{2}$ stock solution calculated by using MaxChelator software (http:// www.stanford.edu/ cpatton/maxc.html). 
At the end of each experiment, the mitochondrial suspension was centrifuged at $9000 \times g\left(10 \mathrm{~min}, 4^{\circ} \mathrm{C}\right)$. The supernatant was discarded and the pellet was immediately frozen and kept in liquid $\mathrm{N}_{2}$ before NMR experiment.

\section{${ }^{1} \mathrm{H}-\mathrm{NMR}$ relaxation studies of mitochondrial water}

Longitudinal (T1) and transversal (T2) proton relaxation times of the mitochondrial pellets, disposed into the Pyrex $10 \times 75$ heavy wall test tubes, were measured on a Bruker NMS 120 minispec ${ }^{\circledR}$ (Wissembourg, France) operating at a Larmor frequency of $20 \mathrm{MHz}$. Thermoregulation was monitored with a Bruker B-VT 2000 variable temperature unit connected to the spectrometer, using nitrogen gas from liquid nitrogen. The sample temperature inside the magnet was determined as described previously. ${ }^{19}$ Relaxation times were measured firstly in the frozen state (structured water fraction, T1sw and T2sw) from 255 to $237 \mathrm{~K}$, on samples removed from liquid nitrogen, and then at $277 \mathrm{~K}$ (total water, T1obs and T2obs). For each sample and each temperature, T1sw were measured in triplicate with 29 to 32 data points (inversion recovery pulse sequence). T2sw were determined with five to 10 successive measurements using a CPMG pulse sequence, with an interpulse delay of $50 \mu \mathrm{s}$, and five to 20 data points. From the temperature dependency of $T 1 s w$ and $T 2 s w$, the rotational and diffusional correlation times of structured water $\tau_{\mathrm{R}}, \tau_{\mathrm{D}}$, and their respective enthalpies $E_{R}$ and $E_{D}$ were calculated (Supplementary text 'Procedure used for the calculation of correlation times $\tau_{D}$ and $\tau_{R}$ '), according to previous studies ${ }^{19}$ and the cross relaxation model of Gallier et al. ${ }^{9}$

The contribution of nonwater protons from residual molecules present in the mitochondrial pellet (mainly mannitol) was evaluated on the basis of the concentration ratio of sugars in the mitochondrial pellet versus supernatant, when the mitochondria are isolated from the liver homogenate with a buffer containing $0.3 \mathrm{M}$ saccharose. The concentration of total sugars present in the samples was determined using the method of Dubois et al. ${ }^{24}$

The exchange process of water molecules between the different compartments was investigated by comparing the relaxation enhancement produced by superparamagnetic magnetite nanoparticles, $10-15 \mathrm{~nm}$ mean hydrodynamic diameter, coated with HEDP (1-hydroxyethylidene diphosphonic acid), ${ }^{25}$ on the bulk (T1obs, T2obs) and structured water phases (Tlsw and T2sw) in the mitochondrial pellet and its supernatant. The contrast agent was added to the first liver homogenate supernatant, after the centrifugation at $1200 \times g$, at the dose of $15 \mathrm{mg} \mathrm{Fe} / \mathrm{ml}$. In the absence of contrast agent, the relaxation processes of bulk water in mitochondrial pellets were also analyzed in the same sample by monoexponential and biexponential fitting, with an interpulse delay of $0.5 \mathrm{~ms}$ and 120 data points (T2obs), and a $5.0 \mathrm{~ms} \times 1.3 \mathrm{IR}$ sequence with 35 points (T10bs).

Equivalent amounts of structured water in mitochondria pellets were calculated from the total magnetization of the samples (height of the NMR signal, $M$, immediately after the $180^{\circ}$ pulse) relative to that of a calibration set of dry bean seeds of different masses and constant water content (12\%) (leguminous seeds were chosen for their low content in lipid bodies, high protein content, and their molecular dynamics of residual water at room temperature comparable to that of unfrozen water in tissues: typical values of $T 1$ and $T 2$ at $20 \mathrm{MHz}$ and room temperature for dry bean seeds are $46 \pm 2$ and $1.30 \pm 0.05 \mathrm{~ms}$, respectively; the analysis of the temperature dependency of their relaxation times shows a pattern comparable to that of frozen liver mitochondria pellets, with $\tau_{\mathrm{R}}$ reaching $5.0 \times 10^{-9} \mathrm{~s}$ around $250 \mathrm{~K}$ ).

\section{Western blots analysis}

Mitochondrial pellets were resuspended in PBS and protein concentrations were determined using the Bradford procedure. Mitochondrial proteins $(10 \mu \mathrm{g})$ were resolved using a $12 \%$ acrylamide/bisacrylamide running gel. Protein transfer (15V, $15 \mathrm{~min})$ was performed using PVDF membranes (Immobilon-P, Millipore, Billerica, MA, USA). Antibodies directed against cytochrome $c$ (dilution: 1/1000; R\&D Systems, Minneapolis, MA, USA), Smac/Diablo (dilution: 1/500; Pro-Sci. Inc., Poway, CA, USA), and FI-ATPase $\beta$-subunit (dilution: 1/1000; Molecular Probes, Eugene, OR, USA) were used for immunoblot analysis. Protein detection was performed using horseradish peroxidase-coupled secondary antibodies (dilution: 1/2000) and ECL-revelation kit (Amersham Biosciences). Signals were quantified using IP Lab Gel software (Signal analytics, Vienna, VA, USA). Quantification values correspond to a ratio cytochrome $c$ or Smac/Diablo signal/FI-ATPase $\beta$-subunit signal.

\section{Statistical analysis}

Paired, two-tailed Student's $t$-test was used to determine the statistical significance of differences between sample means; $0.02<P^{\star}<0.05$, $0.01<P^{\star \star}<0.02,0.001<P^{\star \star \star}<0.01$, and $P^{\star \star \star \star}<0.001$.

\section{Acknowledgements}

This work was supported by grants from INSERM and a special grant from the Ligue Nationale contre le Cancer (Equipe Labellisée Ligue). We thank Pr JP Benoit, Director, for kindly allowing the use of the NMR spectrometer, and $G$ Tanguy for providing the superparamagnetic nanoparticles (Inserm U. 646, 10 rue Andre Boquel, Angers, France). G. Pradal (UFR Medecine, Nantes) is also gratefully acknowledged for TEM micrographs of liver mitochondria.

\section{References}

1. Bernardi $P$, Petronilli V, Di Lisa $F$ and Forte $M$ (2001) A mitochondrial perspective on cell death. Trends Biochem. Sci. 26: 112-117

2. Shi Y (2001) A structural view of mitochondria-mediated apoptosis. Nat. Struct. Biol. 8: 394-401

3. Kroemer G and Reed JC (2000) Mitochondrial control of cell death. Nat. Med. 6: $513-519$

4. Marzo I, Brenner C, Zamzami N, Jürgensmeier JM, Susin SA, Vieira HL, Prévost M-C, Xie Z, Matsuyama S, Reed JC and Kroemer G (1998) Bax and adenine nucleotide translocator cooperate in the mitochondrial control of apoptosis. Science 281: 2027-2031

5. Zamzami $N$ and Kroemer $G$ (2001) The mitochondrion in apoptosis: how Pandora's box opens. Nat. Rev. Mol. Cell. Biol. 2: 67-71

6. Eskes R, Antonsson B, Osen-Sand A, Montessuit S, Richter C, Sadoul R, Mazzei G, Nichols A and Martinou J-C (1998) Bax-induced cytochrome C release from mitochondria is independent of the permeability transition pore but highly dependent on $\mathrm{Mg}^{2+}$ ions. J. Cell Biol. 143: 217-224

7. Martinou JC and Green DR (2001) Breaking the mitochondrial barrier. Nat. Rev. Mol. Cell Biol. 2: 63-67

8. Wüthrich K (1995) Hydration of proteins and nucleic acids in solution. In: NMR in Structural Biology, Wuthrich K (ed) Singapore: World Scientific pp. 621-655

9. Gallier J, Rivet $P$ and de Certaines JD (1987) ${ }^{1} \mathrm{H}$ - and ${ }^{2} \mathrm{H}$-NMR study of bovine serum albumin solutions. Biochim. Biophys. Acta 915: 1-18

10. Hortelano S, Garcia-Martin ML, Cerdan S, Castrillo A, Alvarez AM and Bosca L (2001) Intracellular water motion decreases in apoptotic macrophages after caspase activation. Cell Death Differ. 8: 1022-1028

11. Garlid KD (1979) In: Cell Associated Water, Drost-Hansen W and Clegg J (eds) New York: Academic Press pp. 293-361 
12. Michael $L H$, Seitz $P$, McMillin-Wood J, Chang D, Hazlewood $C$ and Entman $M L$ (1980) Mitochondrial water in myocardial ischemia: Investigation with nuclear magnetic resonance. Science 208: 1267-1269

13. López-Beltrán EA, Maté MJ and Cerdan S (1996) Dynamics and environment of mitochondrial water as detected by ${ }^{1} \mathrm{H}$ NMR. J. Biol. Chem. 271: 1064810653

14. Srere PA (1987) Complexes of sequential metabolic enzymes. Annu. Rev. Biochem. 56: 89-124

15. Fullerton GD (1992) Physiologic basis of magnetic relaxation. In: Magnetic Resonance Imaging, Stark DD, Bradley Jr WG (eds) St Louis: Mosby Year Book pp. 88-108

16. Grieve SM, Wickstead B, Torres AM, Styles P, Wimperis $S$ and Kuchel PW (1998) Multiple-quantum filtered ${ }^{17} \mathrm{O}$ and ${ }^{23} \mathrm{Na} \mathrm{NMR}$ analysis of mitochonrial suspensions. Biophys. Chem. 73: 137-143

17. Klöhn P-C, Soriano ME, Irwin W, Penzo D, Scorrano L, Bitsch A, Neumann H-G and Bernardi $P$ (2003) Early resistance to cell death and to onset of the mitochondrial permeability transition during hepatocarcinogenesis with 2-acetylaminofluorene. Proc. Natl. Acad. Sci. USA 100: 10014-10019

18. Koenig SH, Bryant RG, Hallenga K and Jacob GS (1978) Magnetic crossrelaxation among protons in protein solutions. Biochemistry 17: 4348-4358

19. Pouliquen D, Omnes M-H, Seguin F and Gaignon JL (1998) Changes in the dynamics of structured water and metabolite contents in early developing stages of eggs of turbot (Psetta maxima). Comp. Biochem. Physiol. B 120: 715-726
20. Pouliquen D and Gallois $Y$ (2001) Physicochemical properties of structured water in human albumin and gammaglobulin solutions. Biochimie 83 : 891-898

21. Guihard G, Bellot G, Moreau C, Pradal G, Ferry N, Thorny R, Fichet P, Meflah K and Vallette FM (2004) The mitochondrial-apoptosis induced channel (MAC) corresponds to a late apoptotic event. J. Biol. Chem. 279: 46542-46550

22. Galanos C, Freudenberg MA and Reutter W (1979) Galactosamine-induced sensitization to the lethal effects of endotoxin. Proc. Natl. Acad. Sci. USA 76: 5939-5943

23. Quinlan PT, Thomas AP, Armston AE and Halestrap AP (1983) Measurement of the intramitochondrial volume in hepatocytes without cell disruption and its elevation by hormones and valinomycin. Biochem. J. 214 395-404

24. Dubois M, Gilles KA, Hamilton JK, Rebers PA and Smith F (1956) Colorimetric method for determination of sugars and related substances. Anal. Chem. 28: 350-356

25. Portet D, Denizot B, Rump E, Le Jeune JJ and Jallet $P$ (2001) Nonpolymeric coatings of iron oxide colloids for biological use as magnetic resonance imaging contrast agents. J. Colloid Interface Sci. 238: 37-42

26. Fullerton GD, Potter JL and Dornbluth NC (1982) NMR relaxation of protons in tissues and other macromolecular water solutions. Magn. Reson. Imaging 1 : $209-226$

27. Lundbæk JA, Birn P, Girshman J, Hansen AJ and Andersen OS (1996) Membrane stiffness and channel function. Biochemistry 35: 3825-3830

Supplementary Information accompanies the paper on Cell Death and Differentiation website (http://www.nature.com/cdd) 